\title{
Farnesol inhibits translation to limit growth and filamentation in C. albicans and S. cerevisiae
}

\author{
Nkechi E. Egbe ${ }^{1,2}$, Tawni O. Dornelles ${ }^{1}$, Caroline M. Paget ${ }^{1}$, Lydia M. Castelli ${ }^{1,3}$ and Mark P. Ashe ${ }^{1, *}$ \\ ${ }^{1}$ Division of Molecular and Cellular Function, School of Biological Sciences, Faculty of Biology, Medicine and Health, The University of \\ Manchester, Manchester Academic Health Science Centre, Michael Smith Building, Oxford Rd., Manchester, M13 9PT, United \\ Kingdom. \\ ${ }^{2}$ Current address: Department of Biological Sciences, Nigerian Defence Academy, PMB 2109, Kaduna, Nigeria. \\ ${ }^{3}$ Current address: Sheffield Institute for Translational Neuroscience, University of Sheffield, Sheffield, S1O 2HQ, United Kingdom. \\ * Corresponding Author: \\ Mark P. Ashe, Division of Molecular and Cellular Function, School of Biological Sciences, Faculty of Biology, Medicine and Health, The \\ University of Manchester, Manchester Academic Health Science Centre, Michael Smith Building, Oxford Rd., Manchester, M13 9PT, \\ United Kingdom; Tel: +44 (0)161 306 4164; E-mail: mark.p.ashe@manchester.ac.uk
}

\begin{abstract}
Candida albicans is a polymorphic yeast where the capacity to switch between yeast and filamentous growth is critical for pathogenicity. Farnesol is a quorum-sensing sesquiterpene alcohol that, via regulation of specific signalling and transcription components, inhibits filamentous growth in C. albicans. Here we show that farnesol also inhibits translation at the initiation step in both $C$. albicans and $S$. cerevisiae. In contrast to fusel alcohols, that target the eukaryotic initiation factor 2B (eIF2B), farnesol affects the interaction of the mRNA with the small ribosomal subunit leading to reduced levels of the $48 \mathrm{~S}$ preinitiation ribosomal complex in $S$. cerevisiae. Therefore, farnesol targets a different step in the translation pathway than fusel alcohols to elicit a completely opposite physiological outcome by negating filamentous growth.
\end{abstract}

doi: $10.15698 /$ mic2017.09.589

Received originally: 21.04.2017;

in revised form: 01.08.2017,

Accepted 13.08.2017,

Published 04.09.2017.

Keywords: protein synthesis, translation control, farnesol, quorum sensing.

\section{Abbreviations: \\ QS - quorum sensing, \\ $T C$ - ternary complex.}

\section{INTRODUCTION}

The capacity to detect and respond to environmental change is essential for microorganism survival. This is especially true for opportunist pathogens like Candida albicans; where to initiate infection, the organism must adapt and persist in spite of host immune responses. Typically, $C$. albicans is a harmless commensal, yet in infected patients it causes various different conditions, from mucosal infections to life-threatening systemic infections [1].

Cell-cell signalling, particularly quorum sensing (QS), is a major focus of microbiological research. Farnesol is an acyclic sesquiterpene alcohol that represents the first QS molecule identified in eukaryotic microorganisms [2] where it causes a range of physiological effects [3]. In C. albicans, farnesol inhibits the yeast to hyphal switch [2] to prevent colonization of different niche environments $[4,5]$, it has antioxidant effects [6, 7] and it inhibits transporters [8]. In many species farnesol induces cellular death: for example in the fungal species, Saccharomyces cerevisiae [9], Aspergillus nidulans [10], Penicillium expansum [11], Botrytis cinerea [12] and even $C$. albicans under certain conditions [13]. Equally, farnesol triggers cell death in mammalian cells [14] and can have antibacterial properties $[15,16]$. In fact, farnesol was first discovered as a constituent of plant essential oils with antimicrobial activities [17].

Cellular responses to stimuli act via signal transduction pathways to regulate gene expression. In C. albicans, farnesol targets pathways like the Ras-PKA pathway that, via the transcription factors Efg1p and Czf1p and the repressor Tup1p, regulates gene expression [18]. If a stimulus induces cellular stress, a transient inhibition of global protein synthesis is often observed, which further modulates the programme of gene expression to allow stress responsive gene expression programs to be initiated $[19,20]$. Control of translation in this manner mostly occurs at the initiation stage in order to allow rapid and reversible management of gene expression.

Translation initiation is the assembly of an elongation competent $80 \mathrm{~S}$ ribosome with an initiator methionyl-tRNA (Met-tRNA $_{i}^{\text {Met }}$ ) base paired via its anticodon loop to an mRNA Start codon [21]. Highly conserved controls allow eukaryotic cells to globally reduce translation $[19,20]$ : a prominent example involves elF $2 \alpha$ kinases, like Gcn $2 p$ in $S$. cerevisiae $[22,23]$. Gcn2p activation after amino acid starvation causes phosphorylation of the $\alpha$ subunit of eukaryotic translation initiation factor 2 (elF2) [24]. elF2 is an 
essential GTP-binding protein that interacts with Met$\operatorname{tRNA}_{i}{ }^{\text {Met }}$ to form a ternary complex (TC) that is competent for initiating translation [23]. Phosphorylated elF2 competitively inhibits the elF2B-mediated guanine nucleotide exchange reaction on elF2, reducing TC levels and translation initiation [23]. However, specific mRNAs, such as yeast GCN4, continue to be translated under these conditions. GCN4 encodes a transcription factor that regulates the expression of amino acid biosynthetic genes. This feedback regulatory circuit has proved a paradigm for studies on translation control [25]. Similarly, $C$. albicans expresses a single elF $2 \alpha$ kinase, Gcn2p, which phosphorylates elF $2 \alpha$ in response to various stresses $[26,27]$ and translational activation of CaGCN4 also provides feedback regulation [28, 29].

As well as indirect attenuation of elF2B activity via phosphorylation of elF $2 \alpha$, cells can also modulate elF2B activity more directly. In mammalian cells, phosphorylation of elF2B has been identified as an important regulatory mechanism [30]. In addition, in both yeast and mammalian cells volatile anaesthetics appear to inhibit protein synthesis via elF2B regulation [31, 32]. Moreover, in both $S$. cerevisiae and $C$. albicans, fusel alcohols, which are also characterised as quorum sensing molecules [33], have been shown to inhibit translation initiation in a mechanism that targets elF2B but independently of the Gcn2p kinase or elF2 $\alpha$ phosphorylation [34-36].

Besides control via elF2B, another regulated step in translation initiation is the mRNA selection phase [37]. elF4E and Pab1p select mRNA via interaction with the $5^{\prime}$ cap and $3^{\prime}$ poly $(A)$ tail, respectively. elF4G can interact with both elF4E and Pab1p to form a closed loop complex that, via interactions with elF3, elF5 and elF1, can recruit the small ribosomal subunit to form a $48 \mathrm{~S}$ preinitiation complex [21]. A variety of stress conditions have been shown to target these steps in the initiation pathway leading to transient reductions in translation to facilitate a switch to a new program of gene expression $[19,20]$.

In this study, we show that as well as hampering various filamentation pathways, farnesol inhibits protein synthesis. This inhibition of translation occurs at the initiation step and most likely impacts upon the assembly of the $48 \mathrm{~S}$ preinitiation complex. Intriguingly, this means two different quorum sensing agents, farnesol and fusel alcohols that have conflicting effects on filamentous growth, both inhibit translation initiation but by different mechanisms.

\section{RESULTS AND DISCUSSION}

\section{Farnesol inhibits growth and protein synthesis}

Farnesol, a eukaryotic QS molecule inhibits filamentous growth in both S. cerevisiae and C. albicans [2], however, the concentration required varies according to the specific growth regime [38]. Under the growth conditions used here, we found concentrations in excess of $100 \mu \mathrm{M}$ farnesol inhibited the growth of $C$. albicans and an isogenic gcn2 $2 \Delta$ mutant (Fig. 1A). To study possible origins of the growth inhibition, the impact of farnesol on the rate of protein synthesis was monitored. The resulting $\left[{ }^{35} \mathrm{~S}\right]-$ methionine incorporation data show that farnesol
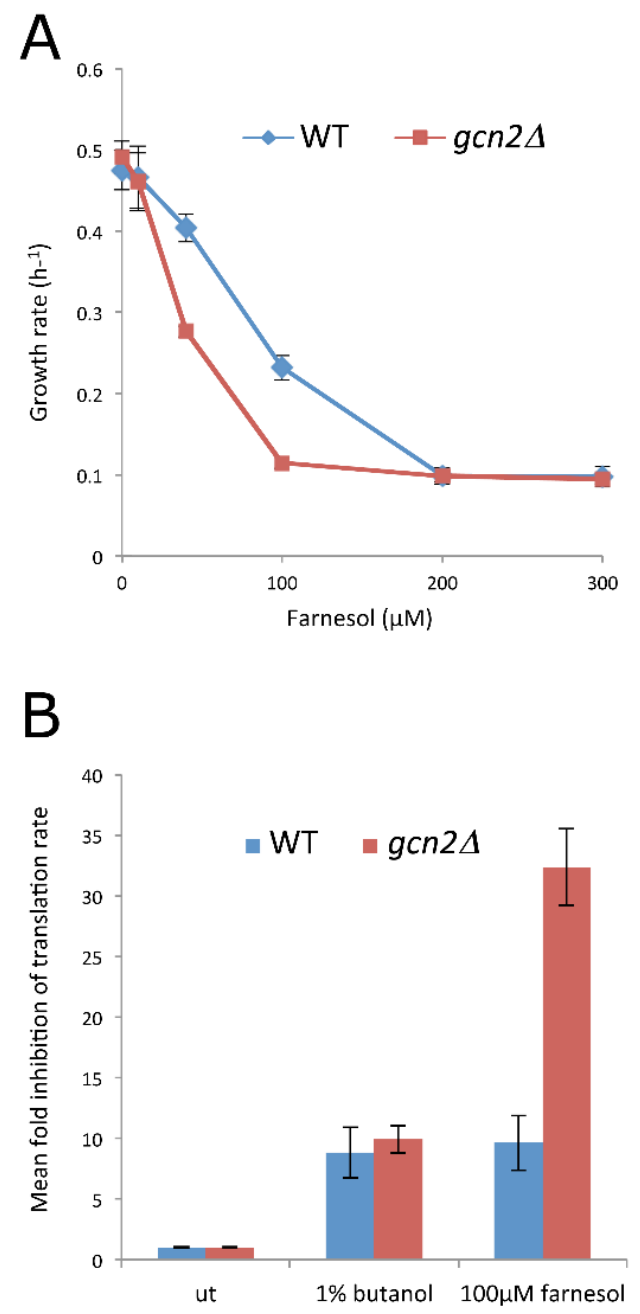

FIGURE 1: Farnesol inhibits growth and protein synthesis. (A) Growth rates for the CAI4 strain and the gcn $2 \Delta$ mutant at various concentrations of farnesol as indicated. (B) Rates of radiolabelled methionine incorporation were measured for the CAI4 strain and the gcn $2 \Delta$ mutant in untreated conditions (ut) or after $1 \%$ butanol or $100 \mu \mathrm{M}$ farnesol treatment. These were used to calculate the fold inhibition for three biological replicates (error bars = SEM).

(300 $\mu \mathrm{M})$ and butanol (2\%) cause a 10 -fold inhibition of protein synthesis in the CAI4 strain of $C$. albicans (Fig. 1B). Therefore, farnesol inhibits protein synthesis at very early stages after addition and this control could contribute to the growth inhibition observed.

To further investigate the stage of protein synthesis that is targeted by farnesol, polysome profiling was used, as this allows both the level of protein synthesis and the stage of regulation to be investigated [34-36]. Analysis of polysome distribution for the $C$. albicans CAI4 strain revealed that increasing concentrations of farnesol caused a change in the polysome profile (Fig. 2A). The $80 \mathrm{~S}$ peak increased dramatically and the polysome peaks were reduced. This change in profile is characteristic of an inhibition of translation initiation [34] and has been observed for many stresses [19]. Similar results in terms of farnesol sensitivity were 
A

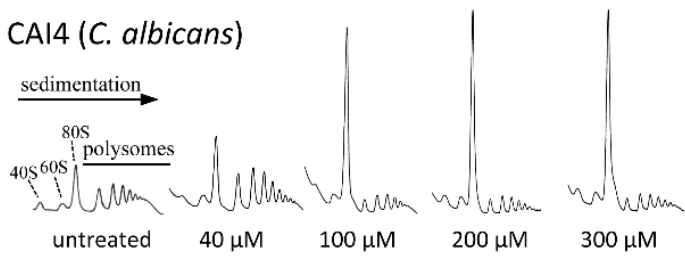

C

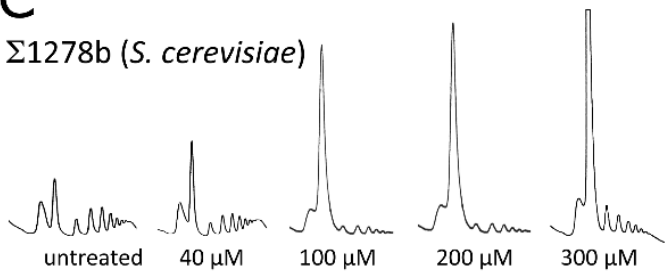

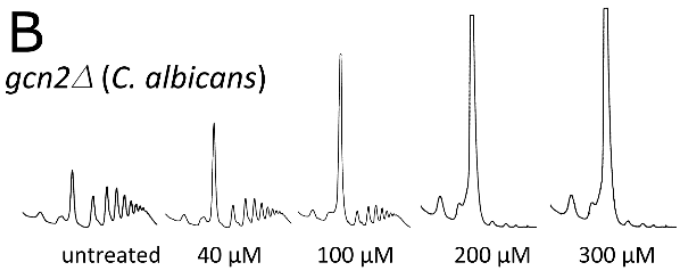

FIGURE 2: Translation initiation is inhibited by farnesol in a Gcn2p-independent manner in $C$. albicans and S. cerevisiae. Figure shows

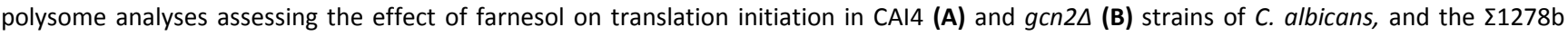
strain of S. cerevisiae (C). Strains were grown in YPD and various concentrations of farnesol were added as indicated for 15 min prior to extract preparation. Extracts were sedimented on $15-50 \%$ sucrose gradients and the absorbance at $254 \mathrm{~nm}$ was continuously measured. The position of $40 \mathrm{~S}, 60 \mathrm{~S}$ and $80 \mathrm{~S}$ peaks are labelled and the direction of sedimentation is noted.

obtained for the $\Sigma 1278 \mathrm{~b}$ strain of S. cerevisiae, where similar concentrations elicited the response across the two yeast species (Fig. 2C). It has been noted previously that the level of free $60 \mathrm{~S}$ is particularly high for the $\Sigma 1278 \mathrm{~b}$, although this does not appear to impact upon its growth or its sensitivity to translational stress [39]. The lowest farnesol concentration that caused a gross impact on polysome distribution for either $C$. albicans or $S$. cerevisiae was $100 \mu \mathrm{M}$ (Fig. 2). This correlated well with the concentration that inhibited growth under the conditions used here (Fig. 1A) suggesting that the inhibition of translation initiation could be intrinsically connected to growth inhibition for farnesol.

The CAI4 strain of C. albicans used here is a commonly used lab strain that is auxotrophic in the uracil biosynthetic pathway by virtue of a homozygous deletion of the URA3 gene [40]. This mutation has previously been shown to alter a number of aspects of $C$. albicans physiology including adhesion and virulence [41]. Therefore, a prototrophic strain of C. albicans, SC5314, was tested. Entirely analogous observations were made on the impact of farnesol on the growth (Fig. S1A) and translation (Fig. S1B) of this strain in response to similar concentrations of farnesol.

Previous studies evaluating QS have established that trans, trans-farnesol is produced by Candida as a QS molecule to inhibit filamentation [2, 42]. Intriguingly, the trans,trans form and mixed stereoisomer preparations both impact upon translation initiation equally (Fig. S1). Indeed $40 \mu \mathrm{M}$ of each is sufficient to induce a mild inhibition of protein synthesis and $100 \mu \mathrm{M}$ leads to a robust inhibition (Fig. 1B). Therefore, in order to explore the mechanism by which farnesol inhibits translation initiation, over the course of the rest of our studies $100 \mu \mathrm{M}$ farnesol was used as this concentration elicits robust inhibition of both growth and translation. However, it should be noted that lower concentrations of farnesol (e.g. $40 \mu \mathrm{M})$ can lead to subtle alterations in the polysome profile (Fig. 2, 3 and S1). This level of sensitivity to farnesol correlates well with earlier studies using similar growth conditions [38].

\section{GCN2 is not involved in the inhibition translation initia-} tion by farnesol

In terms of the mechanism of translational regulation, like S. cerevisiae, $C$. albicans harbours a single elF $2 \alpha$ kinase gene, GCN2, which is involved in the regulation of translation initiation in response to various stresses $[26,27]$. Indeed in S. cerevisiae, gcn2 $\Delta$ mutants are incapable of inhibiting translation initiation in response to specific stress conditions [34], which prevents cells from mounting an appropriate stress response. Therefore, the role of Gcn2p in the farnesol-dependent inhibition of translation initiation was assessed using a gcn $2 \Delta$ mutant strain of $C$. albicans [26]. Previous observations using this strain show that translation initiation remains uninhibited early after amino acid starvation [29]. In terms of the impact of farnesol on growth and translation, the gcn $2 \Delta$ mutant strain is at least as sensitive as the wild type (Fig. $1 \mathrm{~A}$ and $\mathrm{B}$ ). In fact rather than the gcn $2 \Delta$ mutant being resistant to farnesol in terms of translation, as might be expected if the Gcn2p kinase were involved in the control, the farnesol-treated gcn2 $\Delta$ strain is even more inhibited than the wild type. Growth is inhibited at lower farnesol concentrations for the gcn2 $\Delta$ mutant and methionine incorporation is inhibited up to 30 fold (Fig. $1 \mathrm{~A}$ and $\mathrm{B}$ ). Equally, a comparison of the polysome profiles shows that the gcn $2 \Delta$ mutant exhibits somewhat greater sensitivity than the wild type mirroring the growth phenotypes (Fig. 2B). For instance, following treatment with $300 \mu \mathrm{M}$ farnesol, greater polysome run-off is observed for the $g c n 2 \Delta$ mutant compared to the parent strain (cf. Fig. 2B with 2A). Overall, these results show that the 
A
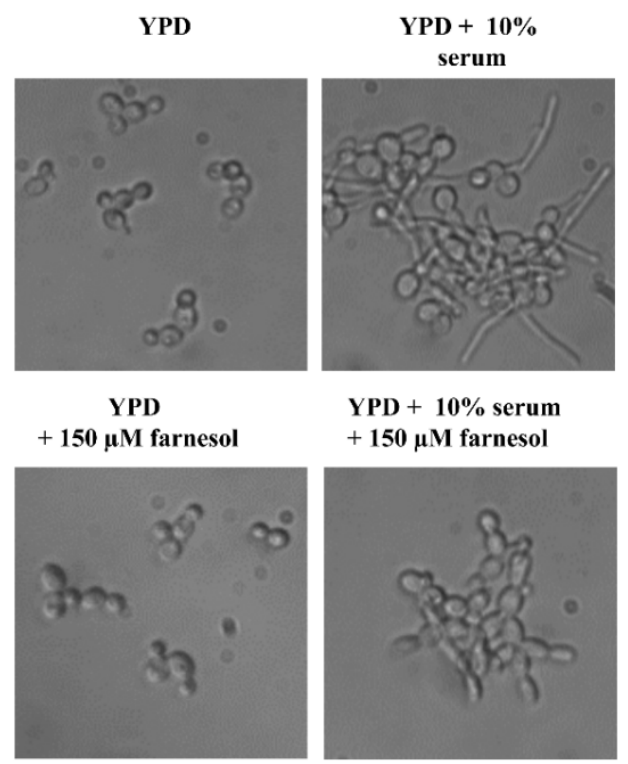

YPD + $10 \%$ serum $+150 \mu M$ farnesol

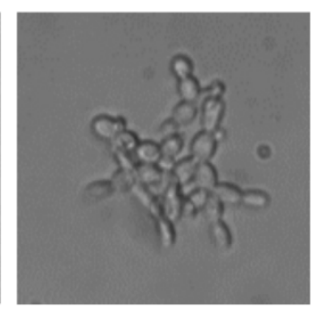

B

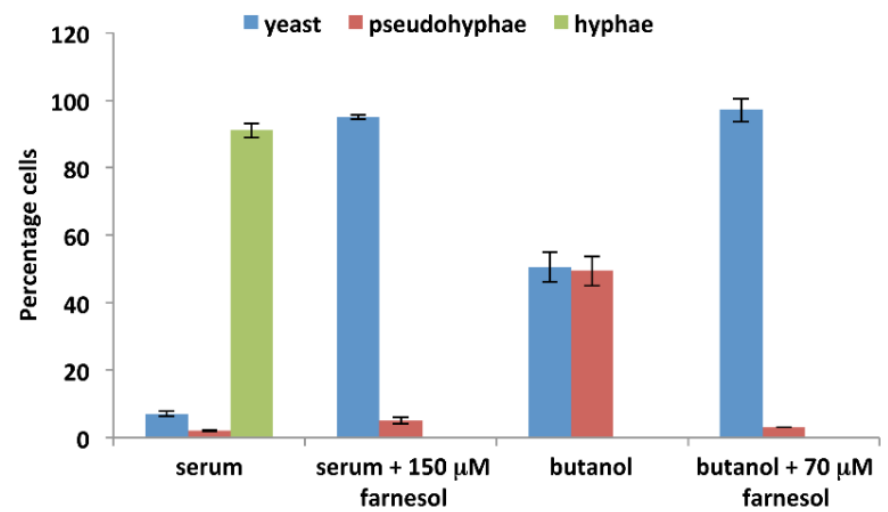

FIGURE 3: Farnesol represses morphological transition in $C$. albicans. (A) Overnight exponential cultures of the CAI4 strain were harvested, washed in water then incubated for 6 hours in different prewarmed media: YPD medium or YPD containing $150 \mu \mathrm{M}$ farnesol; YPD containing $10 \%$ serum or YPD $10 \%$ serum with $150 \mu \mathrm{M}$ farnesol; YPD containing $0.5 \%$ butanol or YPD $0.5 \%$ butanol with $70 \mu \mathrm{M}$ farnesol. Cells were visualised via microscopy and photographed. (B) Cells from the above cultures were counted according to morphology using a cell counting chamber where pseudohyphae were scored if evidence of a restriction point between two cells was apparent, whereas hyphae were scored where elongated cells with no restriction points were viewed. Percentages are an average from three biological replicates (error bars $=$ SEM).
Gcn $2 p$ kinase, that is a requirement for elF $2 \alpha$ phosphorylation and the subsequent regulation of translation initiation in response to a variety of stresses, is not required for the inhibition of translation initiation by farnesol in C. albicans; in fact the gcn2 $\Delta$ mutant is more sensitive to treatment.

Fusel alcohols and other conditions that inhibit protein synthesis in C. albicans promote filamentous growth [36], whereas farnesol inhibits protein synthesis and prevents filamentation. An obvious query is whether the filamentation inducing signal generated by fusel alcohols can be overridden by farnesol or vice versa. Induction of filamentous growth is further complicated as different cues induce distinct forms of filamentation [43]. For instance, fusel alcohols are characterised as inducing pseudohyphal growth [44] where elongated, ellipsoid yeast cells remain attached to one another via constricted septation sites leading to growth of a colony in a branched pattern [45]. In contrast, serum addition elicits true hyphal growth [46], whereby cells are narrow, long, have parallel sides and no obvious constrictions points [45]. In the presence of serum alone over $90 \%$ hyphal growth was observed and the addi- tion of $150 \mu \mathrm{M}$ farnesol blocked the yeast to hyphae switch (Fig. $3 \mathrm{~A}$ and $\mathrm{B}$ ). In contrast, the fusel alcohol, butanol, induces a much less robust effect whereby roughly $50 \%$ of cells exhibit pseudohyphal morphology. Here just $70 \mu \mathrm{M}$ farnesol was sufficient to block any filamentous growth. These results show that farnesol competes with both serum and butanol, but the concentration of farnesol required to effect competition varies according to the strength of the filamentation signal (Fig. $3 \mathrm{~A}$ and B). Curiously, even though both fusel alcohols and farnesol target protein synthesis, they are in competition with respect to their physiological impact on filamentous growth. Thus previous observations suggesting that the inhibition of protein synthesis favours filamentation [36] cannot be generalised across all conditions.

\section{Eukaryotic initiation factor 2B (elF2B) is not regulated by farnesol}

Fusel alcohols inhibit translation initiation in S. cerevisiae and $C$. albicans by targeting the guanine nucleotide exchange factor, elF2B leading to reduced levels of the elF2•GTP•Met-tRNA' ternary complex [34-36]. 
Specific general control response reporters such as the GCN4 reporter mRNA provide a sensitive indicator of changes in the ternary complex and are widely used to study translational regulation [25]. A key observation that pointed towards elF2B as a target for fusel alcohols was the demonstration that these reporters of ternary complex levels are translationally up-regulated [34, 36]. In order to assess this response after farnesol treatment, strains carrying two renilla luciferase reporters were used: the first contains five copies of the general control response element (GCRE), while the second harbours the GCN4 promoter and leader region upstream [28, 29]. Using the parent and gcn $2 \Delta$ mutant strains bearing these reporters, the previous observation that $1 \%$ butanol elicits a non-Gcn $2 p$ dependent increase in the activity of GCRE-LUC and GCN4Luc was confirmed (Fig. $4 \mathrm{~A}$ and B). In stark contrast, farnesol elicits no significant increases of the GCRE-LUC or GCN4LuC reporter expression (Fig. $4 \mathrm{~A}$ and B) suggesting that farnesol does not alter ternary complex levels to activate the GCN response.

Previous studies on the localisation of elF2B in organisms from $S$. cerevisiae to Drosophila melanogaster have defined a large cytoplasmic body called the elF2B body (2B body) [35, 36, 47-49]. Exposure of either $S$. cerevisiae or $C$. albicans cells to fusel alcohols reduces the dynamics of this $2 \mathrm{~B}$ body in a manner that correlates with the sensitivity/ resistance of strains to alcohols $[35,36]$. In order to ascertain whether farnesol also impacts upon 2B body dynamics, a $C$. albicans strain bearing GFP-tagged elF2By was used [36]. Epiflourescence time-lapse microscopy experiments were performed by acquiring images of untreated, butanol treated or farnesol treated cells over a $2 \mathrm{~min}$ period. Movement of the elF2B body across the images was tracked and the total distance $(\mu \mathrm{m})$ moved was calculated. Quantitation of the average displacement shows that $1 \%$ butanol causes total elF2B body movement to drop by
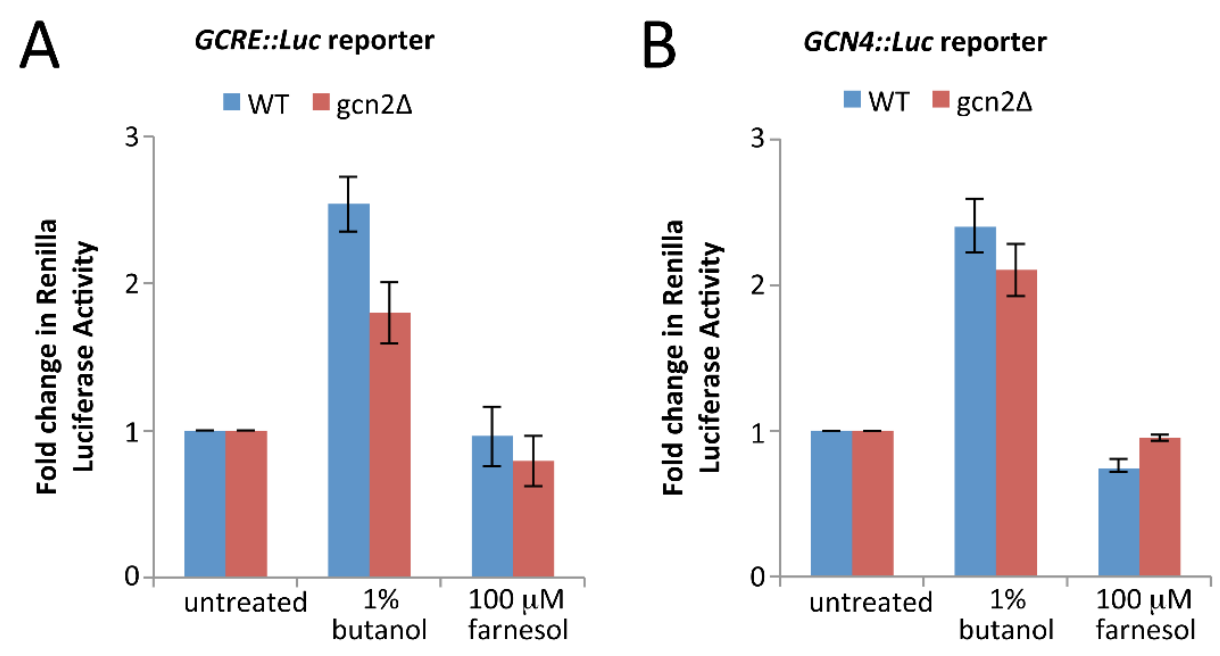

C
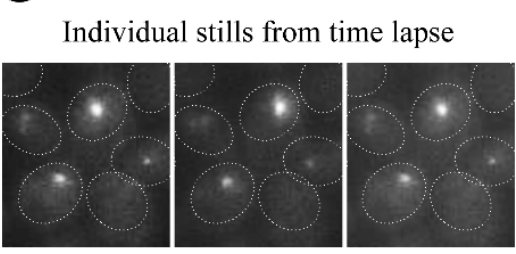

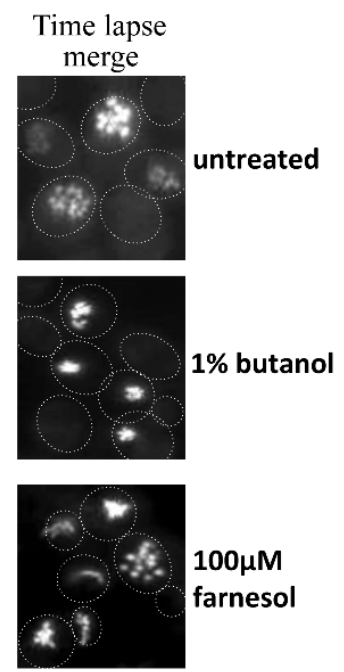

Time lapse merge

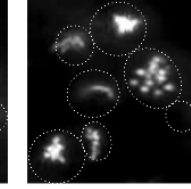

farnesol
D

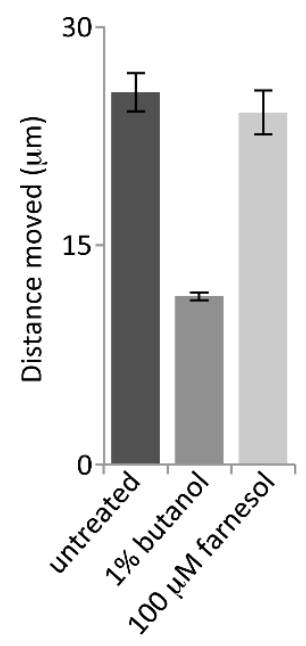

FIGURE 4: Farnesol does not induce GCN4 expression or effect the dynamics of the elF2B body. (A) and (B) CAI4 and gcn2 $\triangle$ strains bearing the GCRE-LUC (A) and GCN4-LuC (B) reporters were treated with $1 \%(\mathrm{v} / \mathrm{v})$ butanol or $100 \mu \mathrm{M}$ farnesol for $2 \mathrm{~h}$, extracts were prepared, then renilla luciferase activity was measured relative to untreated. Error bars $= \pm$ SEM. (C) Images from time-lapse microscopy studies using an elF2B $\gamma$-GFP expressing C. albicans strain. The strains were incubated in media with $1 \%$ butanol, or $100 \mu \mathrm{M}$ farnesol, or they were left untreated (UT) for $15 \mathrm{~min}$ as indicated. Each row contains three stills from a series of 25 images over a period of $2 \mathrm{~min}$, as well as a merged image of all 25 stills, which serves to depict the total extent of 2B body movement. (D) Bar chart depicting the mean distance moved in $\mu$ over a 2-min period from 24 time-lapse experiments. Error bars, \pm 1 SEM.
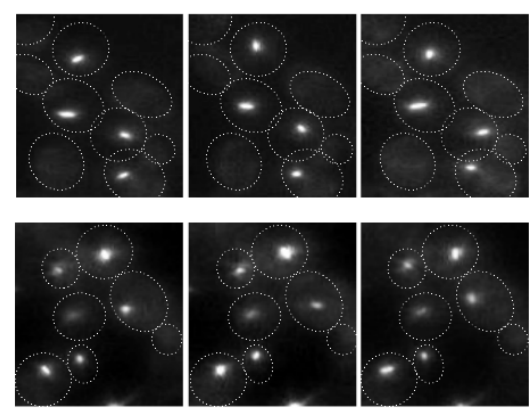
approximately $50 \%$. In contrast, in farnesol treated cells the $2 \mathrm{~B}$ body moves to the same extent as in the untreated cells (Fig. $4 C$ and D). This observation again suggests that the regulatory mechanism by which farnesol inhibits translation initiation is distinct from that of fusel alcohols and is not dependent upon elF2B regulation or the alteration of ternary complex levels.

\section{Farnesol inhibits translation initiation by targeting $48 \mathrm{~S}$ preinitiation complex formation}

Translation initiation is controlled at other levels besides ternary complex formation and elF2B. For instance, the interaction of mRNA with the $43 \mathrm{~S}$ preinitiation complex, i.e. 485 preinitiation complex formation, can also be regulated $[19,20,37]$. This process relies upon interactions between proteins that bind the mRNA and proteins associated with the $40 \mathrm{~S}$ ribosomal subunit complex. One way to assess the factors present with the $40 \mathrm{~S}$ ribosomal subunit is to perform immunoblotting on fractions collected from across sucrose density polysomal gradients. Formaldehyde crosslinking prior to cell lysis stabilizes protein factors in such complexes during the subsequent sedimentation and fractionation steps [50, 51].

A limitation of such studies in C. albicans is that many antibodies against translation factors that are available for S. cerevisiae do not cross-react with $C$. albicans proteins (data not shown). Therefore, to further investigate the step in the translation pathway that is targeted by farnesol, investigations were undertaken in $S$. cerevisiae. The $\Sigma 1278 b$ laboratory strain was selected, as like C. albicans, $\Sigma 1278 \mathrm{~b}$ is diploid and can undergo morphogenetic switching to pseudohyphal growth [44]. In order to validate the
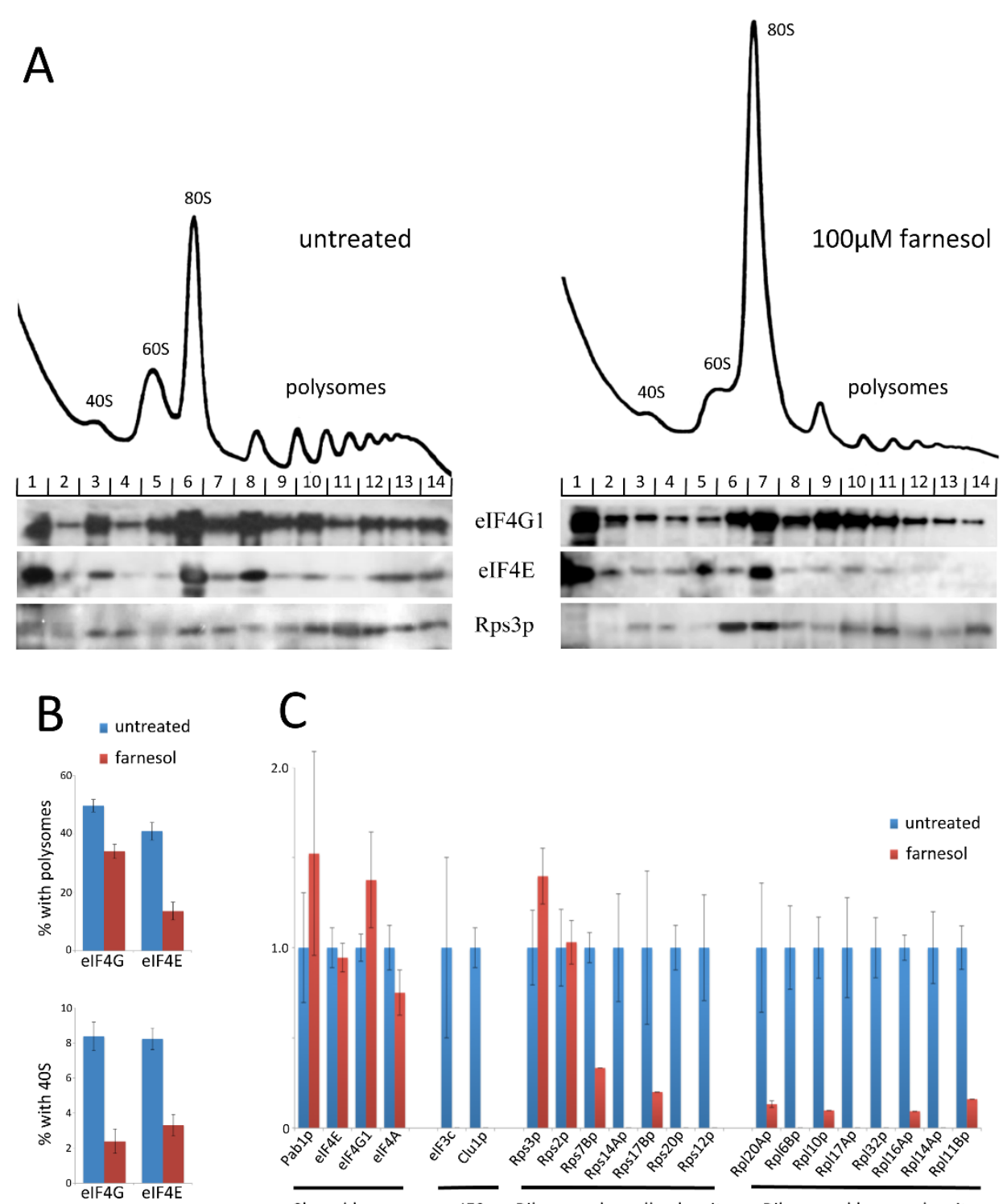
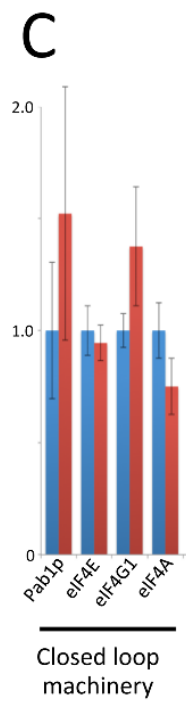

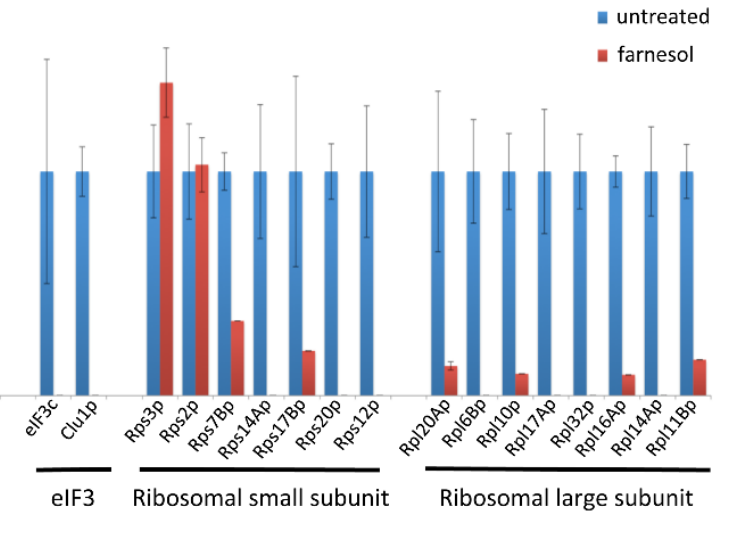

FIGURE 5: Farnesol affects the association of specific translation factors with the 485 pre-ribosomal complex. (A) Formaldehyde sucrose density gradient analysis on extracts from the MLY61 strain either treated with $100 \mu \mathrm{M}$ farnesol for $15 \mathrm{~min}$ or untreated. Immunoblots on gradient fractions are shown below the traces and these were probed using antibodies against the indicated proteins. (B) Quantitation of the proportion of elF4G and elF4E present in the polysome fraction of the gradients before and after farnesol treatment. Quantitation of the proportion of elF4G and elF4E present in the $40 \mathrm{~S}$ region of gradients before and after farnesol treatment. (C) Whole cell extracts and immunoprecipitation samples derived from the elF4G1-TAP tagged strain treated with $100 \mu \mathrm{M}$ farnesol or untreated were analysed via mass spectrometry. The plot shows the relative number of unique peptides that have been matched to the identified protein in the sample where the untreated was normalised to 1 to facilitate a direct comparison of the different translation factors. 
use of this strain, the effects of farnesol on growth and translation were cross-compared. Farnesol inhibits growth and translation initiation over a similar concentration range for the two yeasts and for other lab strains of $S$. cerevisiae, such as BY4741 (Fig. 2C; data not shown), so it seems likely that the translational responses to butanol and farnesol are mechanistically conserved across these species.

Formaldehyde polysome analysis after farnesol treatment revealed an interesting effect in terms of the region of the gradient harbouring the $40 \mathrm{~S}$ ribosomal subunit. This region not only contains free $40 \mathrm{~S}$ ribosomal subunits but also the $48 \mathrm{~S}$ ribosomal preinitiation complex where the $40 \mathrm{~S}$ subunit is associated with mRNA and translation initiation factors i.e. an intermediate in the translation initiation process. A marker for this complex is the presence of translation initiation factors such as elF4E and elF4G that are specifically targeted to the MRNA rather than the $40 \mathrm{~S}$ ribosomal subunit. The level of both elF4G1 and elF4E in the $40 S$ region decreased dramatically after farnesol treatment (Fig. 5A, cf. fraction 3 untreated and fraction 3 treated). Quantitation confirmed that levels dropped from $~ 8-9 \%$ of total to $2-3 \%$ after farnesol treatment (Fig. 5B). Furthermore, both elF4G and elF4E are reduced in polysome regions and this likely reflects reduced levels of initiating ribosomes on mRNAs that are already being translated: although it should be noted that the scale of reduction is greater for elF4E than elF4G (Fig. 5B). This may relate to the fact that elF4G can interact with RNA, Pab1p and other translation factors, whereas elF4E is targeted to the mRNA cap. In sum, these data highlight the possibility that farnesol causes an alteration in protein-protein interactions that lie upstream of $48 \mathrm{~S}$ complex formation.

In order to further investigate how farnesol treatment leads to elF4G/elF4E depletion from regions of the gradient, we undertook an immunopurification-mass spectrometry strategy using an elF4G1-TAP tagged $S$. cerevisiae strain. In terms of the relative number of peptides observed in the immunopurified samples, peptides from known mRNA associated factors, such as elF4G, elF4E, elF4A and Pab1p, were largely unaffected by farnesol (Fig. $5 B)$. In contrast, peptides for other components of the translation machinery were reduced dramatically; including peptides for the ribosomal proteins, as well as subunits of elF3, a translation initiation factor that is associated with the $40 \mathrm{~S}$ ribosomal subunit. Overall, these data support a model where farnesol targets the formation of the $48 \mathrm{~S}$ preinitiation complex to inhibit protein synthesis. The fact that farnesol targets a different step in translation to fusel alcohols, may mean that the impact of these agents and the stage targeted contributes to the opposite effects in terms of filamentous growth (Fig. 3). This is suggestive that translational control plays an important role in the physiological response of $C$. albicans to QS molecules.

\section{DISCUSSION}

A range of alcohols or their derivatives can act as signalling molecules across yeast species [2, 44, 52, 53]. The data presented here combined with that in our previous studies
[36] show that both butanol and farnesol inhibit protein synthesis at the translation initiation stage in C. albicans. Both are metabolites of $C$. albicans that act as signalling molecules, yet have opposing effects on morphological transition [2, 36]. Previously, we have shown in both $S$. cerevisiae and $C$. albicans that short chain alcohols regulate protein synthesis by targeting the guanine nucleotide exchange factor elF2B [34-36].

elF2B regulation plays a critical role in reprograming gene expression as part of the response to stress across different eukaryotic cells [23]. For instance, elF2 phosphorylation by elF $2 \alpha$ kinases, like Gcn $2 p$ in yeast, inhibits elF2B in response to stresses such as amino acid starvation [24], purine starvation [54] and rapamycin treatment [55]. However, while these stresses target elF2B in a Gcn2pdependent manner, the mechanism by which short chain alcohols target elF2B in both S. cerevisiae and C. albicans is Gcn2p-independent [34-36]. The GCN4 reporter experiments and analysis of the elF2B body in this study suggest that the longer chain sesquiterpene alcohol farnesol inhibits translation initiation in a mechanism that does not involve elF2B regulation; either Gcn2p-dependent or independent.

Many studies have reported translational controls targeting steps upstream of $48 \mathrm{~S}$ preinitiation complex formation. For instance, glucose starvation in $S$. cerevisiae causes a reorganisation of the closed loop mRNP translation complex, whereby eIF4A dissociates and the cosedimentation of elF4E, elF4G and Pab1p with ribosomal complexes is compromised [51]. The small non-coding BC RNAs in neuronal cells target the elF4A helicase to inhibit $48 \mathrm{~S}$ preinitiation complex formation on structured mRNAs [56]. Similarly, Burkholderia lethal factor 1, a toxin produced by Burkholderia pseudomallei, which causes the disease melioidosis, provokes a translational block via elF4A [57]. 48S complex formation can also serve as the targeted step when specific mRNAs are translationally regulated. For instance, miRNAs have recently been shown to inhibit target mRNA translation by impacting upon elF4A2 activity [58]. Therefore, a common translational regulatory mechanism that impacts upon the level of the $48 \mathrm{~S}$ preinitiation complex is to target elF4A activity.

In this study, we have investigated how farnesol effects different ribosomal complexes in S. cerevisiae using both formaldehyde-polysome analysis and immunoprecipitation followed by mass spectrometry. Both assays suggest that mRNA-associated translation factors (such as elF4G, elF4E and Pab1p) are associated less well with the ribosome and elF3 following treatment with farnesol. Overall, the observed depletion of elF4G and elF4E from the $40 \mathrm{~S}$ region of polysome gradients combined with the mass spectrometric analysis of elF4G containing complexes lend support to a model where farnesol targets the formation of the $48 \mathrm{~S}$ preinitiation complex to inhibit protein synthesis. This contrasts with the elF2B dependent mechanism by which shorter chain alcohols target translation initiation.

Shorter chain alcohols and farnesol also differ in terms of their effects on morphological transitions in C. albicans. Short chain alcohols induce pseudohyphal growth in 
C. albicans whereas farnesol inhibits this process $[2,44,53]$. Indeed we show that farnesol can impede the filamentation induced by a variety of triggers including short-chain alcohols. This is not without precedent, in C. albicans farnesol also blocks morphogenesis induced by the aromatic alcohol, tyrosol [59]. One intriguing question is how both the shorter chain alcohols and farnesol can target a key ubiquitous process like protein synthesis, yet elicit distinct outcomes in terms of filamentous growth. This question drives at the fundamental physiological rationale for translational regulation in response to changing external conditions. Does the regulation constitute a knee-jerk reaction allowing the preservation of cellular resources by inhibiting the expression of the vast majority of mRNAs, or does the regulation serve a different purpose allowing specific mRNAs to be altered in their translation? In terms of farnesol, we show that translation is mildly inhibited at $40 \mu \mathrm{M}$ farnesol and robustly down-regulated at $100 \mu \mathrm{M}$ farnesol. Various Candida strains produce farnesol up to a concentration of $\sim 60 \mu \mathrm{M}$ [60], which would appear to favour the option where translation of a specific subset of mRNAs is altered. Evidence from a number of systems including the induction of GCN4 translation via amino acid starvation [25] would also favour this option. Under such a scenario, if two stresses impact upon different stages of translation initiation, they might alter the translation of different subsets of mRNA. We have previously observed evidence for such effects in S. cerevisiae, where fusel alcohols and amino acid starvation alter translational reprograming to allow continued translation of different cohorts of mRNAs [61]. With this in mind, we envisage that for farnesol and fusel alcohols, mRNAs encoding pro and antifilamentation factors might be prominent in a set that are differentially regulated at the translational level. Such effects would also be integrated with well-defined transcriptional controls, especially for farnesol [18], to produce very different phenotypic outcomes.

\section{MATERIALS AND METHODS \\ Media and growth conditions}

The strains in Table 1 were grown and maintained as described previously [36]. Butanol and farnesol were routinely added for $15 \mathrm{~min}$ at the concentrations stated. Unless otherwise stated trans,trans-farnesol was used. Tolerance was assessed by adding butanol $(0.5 \%, 1 \%$ and $2 \%)$ or farnesol ( $40 \mu \mathrm{M}, 100 \mu \mathrm{M}, 200 \mu \mathrm{M}$ and $300 \mu \mathrm{M})$ to strains at $\mathrm{OD}_{600} 0.1$ and then testing growth.

\section{Morphogenesis assays}

Exponential cultures were harvested, washed in water then re-inoculated into media with $0.5 \%$ butanol, $10 \%$ serum, $0.5 \%$ butanol - $70 \mu \mathrm{M}$ farnesol or $10 \%$ serum - $150 \mu \mathrm{M}$ farnesol. Filamentation was assessed microscopically as previously described [36].

\section{Analysis of polysomes and other translation assays}

Exponential strains were incubated with butanol/ farnesol for $15 \mathrm{~min}$ then treated with cycloheximide: $1 \mathrm{mg} / \mathrm{ml}$ (C. albicans) or $0.1 \mathrm{mg} / \mathrm{ml}$ (S. cerevisiae). Extracts were prepared then polysome analysis and fractionation were

TABLE 1. Strains used in this study.

\begin{tabular}{|c|c|c|}
\hline \multicolumn{3}{|c|}{ C. albicans strains } \\
\hline Strain & Genotype & Source \\
\hline CAI4 & 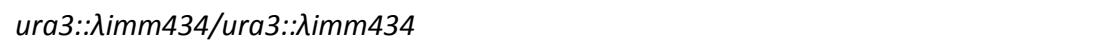 & A. Brown \\
\hline CAI8 & 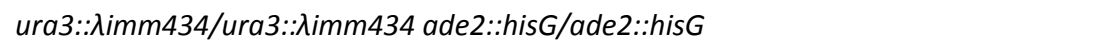 & A. Brown \\
\hline 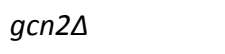 & 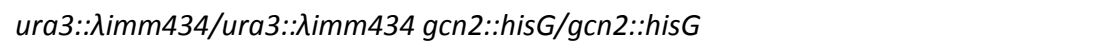 & A. Brown \\
\hline SC5314 & Prototroph & C. Grant \\
\hline уМК2313 & 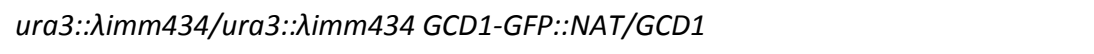 & Ashe lab \\
\hline CY2383 & ura3::גimm434/ura3::גimm434 ADE2::GCRE-rLUC/ade2::hisG & C. Grant \\
\hline CY2511 & 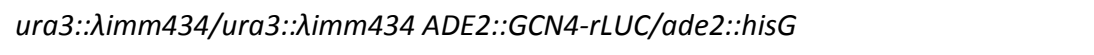 & C. Grant \\
\hline CY2387 & ura3::גimm434/ura3::גimm434 gcn2::hisG/gcn2::hisG ADE2::GCRE-rLUC/ade2::hisG & C. Grant \\
\hline CY2492 & ura3::גimm434/ura3::גimm434 gcn2::hisG/gcn2::hisG ADE2::GCN4-rLUC/ade2::hisG & C. Grant \\
\hline \multicolumn{3}{|c|}{ S. cerevisiae strains } \\
\hline Strain & Genotype & Source \\
\hline $\operatorname{MLY} 61(\Sigma 1278 b)$ & MATa/MAT $\alpha$ ura3-52/ura3-52 & J. Heitman \\
\hline уМК2197 & MATa his3 $\Delta 1$ leu2 20 met $15 \Delta O$ ura3 $\Delta O$ HIS3 & Open biosystems \\
\hline yMK2084 & 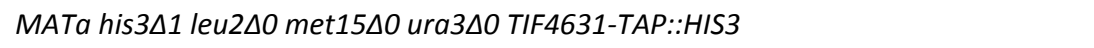 & Open Biosystems \\
\hline
\end{tabular}


carried out as previous [36]. Formaldehyde polysome analysis was performed as described previously [50,51]. Immunoblots were probed with antibodies to yeast elF4G, eIF4E and Rps3.

$\left[{ }^{35} \mathrm{~S}\right]$-methionine incorporation assays were conducted by adding $60 \mathrm{ng} / \mathrm{ml}$ methionine, where $0.5 \mathrm{ng} / \mathrm{ml}$ was $\left[{ }^{35} \mathrm{~S}\right]-$ methionine (PerkinElmer), to exponential untreated or farnesol/ butanol treated cultures in synthetic complete dextrose $(\mathrm{SCD})$ medium lacking methionine. Samples (1 ml) were taken at the indicated times and processed as described previously [36].

For the Luciferase reporter assays [29], lysates were prepared from exponential untreated or farnesol/ butanol treated cultures in RLUC buffer $\left(0.5 \mathrm{M} \mathrm{NaCl}, 0.1 \mathrm{M} \mathrm{K}_{2} \mathrm{HPO}_{4}\right.$, $1 \mathrm{mM} \mathrm{Na}{ }_{2}$ EDTA, $0.6 \mathrm{mM}$ sodium azide, $1 \mathrm{mM}$ phenylmethylsulfonyl fluoride, $0.02 \%$ bovine serum albumin). $1.25 \mu \mathrm{M}$ coelentrazine $\mathrm{h}$ (Promega) was added to the extracts to initiate the reaction, and activity was measured using a GloMax 20/20 luminometer (Promega). Luciferase activity (RLU) is expressed as relative luminescence per $10 \mathrm{~s} / \mathrm{mg}$ protein.

For studies on the 2B-body [35, 36], real-time 2D deconvolved projections were generated via continuous z-sweep acquisition on a Delta Vision RT microscope (Applied Precision, Isaaquah, WA) with an Olympus $100 \times 1.40$ NA DIC oil PlanApo objective (Melville, NY) and Roper CoolSnap HQ camera (Tucson, AZ) with Applied Precision Softworx 1.1 software for fast visualisation of all planes with minimal fluorescent bleaching. Images were acquired every $5 \mathrm{~s}$ over a 2 min period, and ImageJ (http://rsb.info.nih.gov/ij/; NIH) was used to track $2 \mathrm{~B}$ body movement and calculate the mean total distance using at least 24 individual tracking experiments per condition.

\section{Affinity Purification and mass spectrometry}

For the elF4G1-TAP purification, protein extracts were bound to IgG columns eluted with a TAP peptide, then samples were isolated from SDS PAGE gel slices [62]. Dried gel pieces containing the whole protein sample were digested using $100 \mathrm{ng}$ trypsin and analysed by LC-MS/MS using an UltiMate ${ }^{\circledR} 3000$ Rapid Separation liquid chromatography (Dionex Corporation, Sunnyvale, CA) coupled to a LTQ Velos Pro mass spectrometer (Thermo Fisher Scientific,
Waltham, MA). Data were searched using Mascot (Matrix Science UK), against the Uniprot database with S. cerevisiae selected. Data were validated and further processed using Scaffold (Proteome Software, Portland, OR).

\section{ACKNOWLEDGEMENTS}

We thank A. Brown (Aberdeen University), C. Grant (University of Manchester (UoM)) and M. Pool (UoM) for strains and antibodies. We thank P. March and S. Mardsen for their help with the microscopy. The Bioimaging Facility microscopes used rely on grants by the Biotechnology and Biological Sciences Research Council (BBSRC), the Wellcome Trust and a UoM Strategic Fund. We are grateful to David Knight and Emma-Jane Keevil in the Biomolecular Analysis Core Facility, The University of Manchester for their help with mass spectrometry analysis. NEE was funded by the Tertiary education trust fund Nigeria, TD was funded by the Coordenação de Aperfeiçoamento de Pessoal de Nível Superior (CAPES)- science without borders program, CMP was funded by a BBSRC project grant (BB/K002767/1) and LMC was funded by a BBSRC LoLa grant (BB/G012571/1).

\section{SUPPLEMENTAL MATERIAL}

All supplemental data for this article are available online at www.microbialcell.com.

\section{CONFLICT OF INTEREST}

The authors declare no conflict of interest.

\section{COPYRIGHT}

(C) 2017 Egbe et al. This is an open-access article released under the terms of the Creative Commons Attribution (CC BY) license, which allows the unrestricted use, distribution, and reproduction in any medium, provided the original author and source are acknowledged.

Please cite this article as: Nkechi E. Egbe, Tawni O. Dornelles, Caroline M. Paget, Lydia M. Castelli and Mark P. Ashe (2017). Farnesol inhibits translation to limit growth and filamentation in C. albicans and S. cerevisiae. Microbial Cell 4(9): 294-304. doi: 10.15698/mic2017.09.589

\section{REFERENCES}

1. Mayer FL, Wilson D, Hube B (2013). Candida albicans pathogenicity mechanisms. Virulence 4(2): 119-128.

2. Hornby JM, Jensen EC, Lisec AD, Tasto JJ, Jahnke B, Shoemaker R, Dussault $P$, Nickerson KW (2001). Quorum sensing in the dimorphic fungus Candida albicans is mediated by farnesol. Appl Environmental Microbiol 67(7): 2982-2992.

3. Langford ML, Atkin AL, Nickerson KW (2009). Cellular interactions of farnesol, a quorum-sensing molecule produced by Candida albicans. Future Microbiol 4(10): 1353-1362.

4. Ramage G, Saville SP, Wickes BL, Lopez-Ribot JL (2002). Inhibition of Candida albicans biofilm formation by farnesol, a quorum-sensing molecule. Appl Environmental Microbiol 68(11): 5459-5463.
5. Martins M, Henriques M, Azeredo J, Rocha SM, Coimbra MA Oliveira R (2007). Morphogenesis control in Candida albicans and Candida dubliniensis through signaling molecules produced by planktonic and biofilm cells. Eukaryot Cell 6(12): 2429-2436.

6. Westwater C, Balish E, Schofield DA (2005). Candida albicansconditioned medium protects yeast cells from oxidative stress: a possible link between quorum sensing and oxidative stress resistance. Eukaryot Cell 4(10): 1654-1661.

7. Deveau A, Piispanen AE, Jackson AA, Hogan DA (2010). Farnesol induces hydrogen peroxide resistance in Candida albicans yeast by inhibiting the Ras-cyclic AMP signaling pathway. Eukaryot Cell 9(4): 569-577. 
8. Sharma M, Prasad R (2011). The quorum-sensing molecule farnesol is a modulator of drug efflux mediated by $A B C$ multidrug transporters and synergizes with drugs in Candida albicans. Antimicrob Agents Chemother 55(10): 4834-4843.

9. Machida K, Tanaka T, Yano Y, Otani S, Taniguchi M (1999). Farnesolinduced growth inhibition in Saccharomyces cerevisiae by a cell cycle mechanism. Microbiology 145(Pt 2): 293-299.

10. Dinamarco TM, Goldman MH, Goldman GH (2011). Farnesolinduced cell death in the filamentous fungus Aspergillus nidulans. Biochem Soc Trans 39(5): 1544-1548.

11. Liu P, Luo L, Guo J, Liu H, Wang B, Deng B, Long CA, Cheng Y (2010). Farnesol induces apoptosis and oxidative stress in the fungal pathogen Penicillium expansum. Mycologia 102(2): 311-318.

12. Cotoras M, Castro P, Vivanco H, Melo R, Mendoza L (2013). Farnesol induces apoptosis-like phenotype in the phytopathogenic fungus Botrytis cinerea. Mycologia 105(1): 28-33.

13. Shirtliff ME, Krom BP, Meijering RA, Peters BM, Zhu J, Scheper MA, Harris ML, Jabra-Rizk MA (2009). Farnesol-induced apoptosis in Candida albicans. Antimicrob Agents Chemother 53(6): 2392-2401.

14. Joo JH, Jetten AM (2010). Molecular mechanisms involved in farnesol-induced apoptosis. Cancer lett 287(2): 123-135.

15. Gomes Fl, Teixeira P, Azeredo J, Oliveira R (2009). Effect of farnesol on planktonic and biofilm cells of Staphylococcus epidermidis. Curr Microbiol 59(2): 118-122.

16. Jabra-Rizk MA, Meiller TF, James CE, Shirtliff ME (2006). Effect of farnesol on Staphylococcus aureus biofilm formation and antimicrobial susceptibility. Antimicrob Agents Chemother 50(4): 1463-1469.

17. Kuete V, Efferth $T$ (2013). Molecular determinants of cancer cell sensitivity and resistance towards the sesquiterpene farnesol. Pharmazie 68(7): 608-615.

18. Langford ML, Hargarten JC, Patefield KD, Marta E, Blankenship JR, Fanning S, Nickerson KW, Atkin AL (2013). Candida albicans Czf1 and Efg1 coordinate the response to farnesol during quorum sensing, white-opaque thermal dimorphism, and cell death. Eukaryot cell 12(9): 1281-1292.

19. Simpson CE, Ashe MP (2012). Adaptation to stress in yeast: to translate or not? Biochem Soc Trans 40(4): 794-799.

20. Spriggs KA, Bushell M, Willis AE (2010). Translational regulation of gene expression during conditions of cell stress. Mol Cell 40(2): 228237.

21. Hinnebusch AG (2014). The scanning mechanism of eukaryotic translation initiation. Ann Rev Biochem 83: 779-812.

22. Pavitt GD (2005). elF2B, a mediator of general and gene-specific translational control. Biochem Soc Trans 33(Pt 6): 1487-1492.

23. Wek RC, Jiang HY, Anthony TG (2006). Coping with stress: elF2 kinases and translational control. Biochem Soc Trans 34(Pt 1): 7-11.

24. Dever TE, Feng L, Wek RC, Cigan AM, Donahue TF, Hinnebusch AG (1992). Phosphorylation of initiation factor 2 alpha by protein kinase GCN2 mediates gene-specific translational control of GCN4 in yeast. Cell 68(3): 585-596.

25. Hinnebusch AG (2005). Translational regulation of GCN4 and the general amino acid control of yeast. Annu Rev Microbiol 59: 407-450.

26. Tournu H, Tripathi G, Bertram G, Macaskill S, Mavor A, Walker L, Odds FC, Gow NA, Brown AJ (2005). Global role of the protein kinase $\mathrm{Gcn} 2$ in the human pathogen Candida albicans. Eukaryot cell 4(10): 1687-1696.
27. Sundaram A, Grant CM (2014). Oxidant-specific regulation of protein synthesis in Candida albicans. Fungal Genet Biol 67: 15-23.

28. Tripathi G, Wiltshire C, Macaskill S, Tournu H, Budge S, Brown AJ (2002). Gcn 4 co-ordinates morphogenetic and metabolic responses to amino acid starvation in Candida albicans. EMBO J 21(20): 5448-5456.

29. Sundaram A, Grant CM (2014). A single inhibitory upstream open reading frame (UORF) is sufficient to regulate Candida albicans GCN4 translation in response to amino acid starvation conditions. RNA 20(4): 559-567.

30. Wang X, Paulin FE, Campbell LE, Gomez E, O'Brien K, Morrice N, Proud CG (2001). Eukaryotic initiation factor 2B: identification of multiple phosphorylation sites in the epsilon-subunit and their functions in vivo. EMBO J 20(16): 4349-4359.

31. Palmer LK, Rannels SL, Kimball SR, Jefferson LS, Keil RL (2006). Inhibition of mammalian translation initiation by volatile anesthetics. Am J Physiol Endocrinol Metab 290(6): E1267-E1275.

32. Palmer LK, Shoemaker JL, Baptiste BA, Wolfe D, Keil RL (2005). Inhibition of translation initiation by volatile anesthetics involves nutrient-sensitive GCN-independent and -dependent processes in yeast. Mol Biol Cell 16(8): 3727-3739.

33. Hogan DA (2006). Quorum sensing: alcohols in a social situation. Curr Biol 16(12): R457-458.

34. Ashe MP, Slaven JW, De Long SK, Ibrahimo S, Sachs AB (2001). A novel elF2B-dependent mechanism of translational control in yeast as a response to fusel alcohols. EMBO J 20(22): 6464-6474.

35. Taylor EJ, Campbell SG, Griffiths CD, Reid PJ, Slaven JW, Harrison RJ, Sims PF, Pavitt GD, Delneri D, Ashe MP (2010). Fusel alcohols regulate translation initiation by inhibiting elF2B to reduce ternary complex in a mechanism that may involve altering the integrity and dynamics of the elF2B body. Mol Biol Cell 21(13): 2202-2216.

36. Egbe NE, Paget CM, Wang H, Ashe MP (2015). Alcohols inhibit translation to regulate morphogenesis in C. albicans. Fungal Genet Biol 77: 50-60.

37. Richter JD, Sonenberg N (2005). Regulation of cap-dependent translation by elF4E inhibitory proteins. Nature 433(7025): 477-480.

38. Langford ML, Hasim S, Nickerson KW, Atkin AL (2010). Activity and toxicity of farnesol towards Candida albicans are dependent on growth conditions. Antimicrob Agents Chemother 54(2): 940-942.

39. Ibrahimo S, Holmes LE, Ashe MP (2006). Regulation of translation initiation by the yeast elF4E binding proteins is required for the pseudohyphal response. Yeast 23(14-15): 1075-1088.

40. Fonzi WA, Irwin MY (1993). Isogenic strain construction and gene mapping in Candida albicans. Genetics 134(3): 717-728.

41. Staab JF, Sundstrom $P$ (2003). URA3 as a selectable marker for disruption and virulence assessment of Candida albicans genes. Trends Microbiol 11(2): 69-73.

42. Shchepin R, Hornby JM, Burger E, Niessen T, Dussault P, Nickerson KW (2003). Quorum sensing in Candida albicans: probing farnesol's mode of action with 40 natural and synthetic farnesol analogs. Chem Biol 10(8): 743-750.

43. Berman J (2006). Morphogenesis and cell cycle progression in Candida albicans. Curr Opin Microbiol 9(6): 595-601.

44. Lorenz MC, Cutler NS, Heitman J (2000). Characterization of alcohol-induced filamentous growth in Saccharomyces cerevisiae. Mol Biol Cell 11(1): 183-199.

45. Sudbery P, Gow N, Berman J (2004). The distinct morphogenic states of Candida albicans. Trends Microbiol 12(7): 317-324. 
46. Ogletree FF, Abdelal AT, Ahearn DG (1978). Germ-tube formation by atypical strains of Candida albicans. Antonie van Leeuwenhoek 44(1): 15-24.

47. Campbell SG, Hoyle NP, Ashe MP (2005). Dynamic cycling of elF2 through a large elF2B-containing cytoplasmic body: implications for translation control. J Cell Biol 170(6): 925-934.

48. Browne CM, Samir P, Fites JS, Villarreal SA, Link AJ (2013). The yeast eukaryotic translation initiation factor $2 \mathrm{~B}$ translation initiation complex interacts with the fatty acid synthesis enzyme YBR159W and endoplasmic reticulum membranes. Mol Cell Biol 33(5): 1041-1056.

49. Noree C, Sato BK, Broyer RM, Wilhelm JE (2010). Identification of novel filament-forming proteins in Saccharomyces cerevisiae and Drosophila melanogaster. J Cell Biol 190(4): 541-551.

50. Nielsen KH, Szamecz B, Valasek L, Jivotovskaya A, Shin BS, Hinnebusch AG (2004). Functions of elF3 downstream of $48 \mathrm{~S}$ assembly impact AUG recognition and GCN4 translational control. EMBO J 23(5): 1166-1177.

51. Castelli LM, Lui J, Campbell SG, Rowe W, Zeef LAH, Holmes LEA, Hoyle NP, Bone J, Selley JN, Sims PFG, Ashe MP (2011). Glucose depletion inhibits translation initiation via elF4A loss and subsequent $48 \mathrm{~S}$ preinitiation complex accumulation, while the pentose phosphate pathway is coordinately up-regulated. Mol Biol Cell 22(18): 33793393.

52. Chen H, Fink GR (2006). Feedback control of morphogenesis in fungi by aromatic alcohols. Genes Dev 20(9): 1150-1161.

53. Dickinson JR (1996). 'Fusel' alcohols induce hyphal-like extensions and pseudohyphal formation in yeasts. Microbiology 142(Pt 6): 13911397.

54. Rolfes RJ, Hinnebusch AG (1993). Translation of the yeast transcriptional activator GCN4 is stimulated by purine limitation: implications for activation of the protein kinase GCN2. Mol Cell Biol 13(8): 5099-5111.
55. Kubota H, Obata T, Ota K, Sasaki T, Ito T (2003). Rapamycininduced translational derepression of GCN4 mRNA involves a novel mechanism for activation of the elF2 alpha kinase GCN2. J Biol Chem 278(23): 20457-20460.

56. Eom T, Berardi V, Zhong J, Risuleo G, Tiedge H (2011). Dual nature of translational control by regulatory BC RNAs. Mol Cell Biol 31(22): 4538-4549.

57. Cruz-Migoni A, Hautbergue GM, Artymiuk PJ, Baker PJ, BokoriBrown M, Chang CT, Dickman MJ, Essex-Lopresti A, Harding SV, Mahadi NM, Marshall LE, Mobbs GW, Mohamed R, Nathan S, Ngugi SA, Ong C, Ooi WF, Partridge LJ, Phillips HL, Raih MF, Ruzheinikov S, Sarkar-Tyson M, Sedelnikova SE, Smither SJ, Tan P, Titball RW, Wilson SA, Rice DW (2011). A Burkholderia pseudomallei toxin inhibits helicase activity of translation factor elF4A. Science 334(6057): 821 824.

58. Meijer HA, Kong YW, Lu WT, Wilczynska A, Spriggs RV, Robinson SW, Godfrey JD, Willis AE, Bushell M (2013). Translational repression and elF4A2 activity are critical for microRNA-mediated gene regulation. Science 340(6128): 82-85.

59. Ghosh S, Kebaara BW, Atkin AL, Nickerson KW (2008). Regulation of aromatic alcohol production in Candida albicans. Appl Environmental Microbiol 74(23): 7211-7218.

60. Weber K, Sohr R, Schulz B, Fleischhacker M, Ruhnke M. (2008). Secretion of E,E-farnesol and biofilm formation in eight different Candida species. Antimicrob Agents Chemother 52(5): 1859-61.

61. Smirnova JB, Selley JN, Sanchez-Cabo F, Carroll K, Eddy AA, McCarthy JE, Hubbard SJ, Pavitt GD, Grant CM, Ashe MP (2005). Global gene expression profiling reveals widespread yet distinctive translational responses to different eukaryotic translation initiation factor 2B-targeting stress pathways. Mol Cell Biol 25(21): 9340-9349.

62. Castelli LM, Talavera D, Kershaw CJ, Mohammad-Qureshi SS, Costello JL, Rowe W, Sims PF, Grant CM, Hubbard SJ, Ashe MP, Pavitt GD (2015). The 4E-BP Caf20p Mediates Both elF4E-Dependent and Independent Repression of Translation. PLoS Genet 11(5): e1005233. 